\title{
Group B Streptococcus Recto-Vaginal Colonization in Near-Term Pregnant Women, Southwest Ethiopia
}

\author{
Woubishet Girma $^{1^{*}}$, Nadia Yimer ${ }^{2}$, Tesfaye Kassa ${ }^{3}$, Elias Yesuf ${ }^{4,5}$
}

\section{OPEN ACCESS}

Citation: Girma, Woubishet, Yimer, Nadia, Kassa Tesfaye, Yesuf Elias. Group B Streptococcus Recto-Vaginal Colonization in Near-Term Pregnant Women, Southwest Ethiopia. Ethiop J Health Sci. 2020;30(5):687. doi:http:// dx.doi.org/ 10.4314/ejhs.v30i5.7

Received: March 13, 2020

Accepted: May 09, 2020

Published: September 1, 2020

Copyright: (C) 2020 Girma Woubishet, et al. This is an open access article distributed under the terms of the Creative Commons Attribution License, which permits unrestricted use, distribution, and reproduction in any medium, provided the original author and source are credited. Funding: Nil

Competing Interests: The authors declare that this manuscript was approved by all authors in its form and that no competing interest exists.

Affiliation and Correspondence:

${ }^{1 *}$ Corresponding Author, Obstetrician and gynecologist, Department of obstetrics and gynecology, Jimma Institute of health sciences, Jimma, Ethiopia, Email: gwubdz@yahoo.com

${ }^{2}$ Obstetrician and gynecologist, private hospital, Dessie, Ethiopia, E-mail: nadiayimer@yahoo.com ${ }^{3}$ School of Medical Laboratory, Jimma University Institute of Health, Jimma, Ethiopia. E-mail: ktes36@gmail.com

${ }^{4}$ Department of Health Economics, Management, and Policy; Jimma University Institute of Health; Jimma, Ethiopia

${ }^{5} \mathrm{CIH}^{\mathrm{LMU}}$ Center for International Health, University of Munich, Munich, Germany.

E-mail: gwubdz@yahoo.com

\section{ABSTRACT}

BACKGROUND: Group B Streptococcus (GBS) is recognized as an important cause of maternal and neonatal morbidity and mortality. Maternal vaginal carriage of GBS (Streptococcus agalactiae) can lead to vertical transmission to the neonate at the time of delivery. However, little is known about its prevalence, predictors and antibiotic susceptibility pattern in Jimma, Ethiopia. This study assessed the prevalence, antimicrobial susceptibility pattern and determinants of GBS recto-vaginal colonization among near-term pregnant women.

METHODS: A cross-sectional study was conducted from May to August 2015 at Jimma University Medical Centre in Southwest Ethiopia. Data through questionnaire and GBS isolates from vaginal and rectal swabs were collected. Antimicrobial susceptibility testing was performed.

RESULTS: The overall prevalence of GBS colonization among near term pregnant women (35-37 weeks) was $16.3 \%$ (22/135). The majority of GBS isolates were sensitive to Ampicillin and Penicillin $G$ with $95.5 \%$ and $90.1 \%$, respectively. Erythromycin and clindamycin were resisted by $50 \%$ and $40.9 \%$ of the isolates, respectively, whereas gentamicin was resisted by all isolates. GBS colonization was significantly associated with a history of preterm delivery (PTD) (AOR: 6.3, 95\% CI: 1.42, 28.3) and history of urinary tract infection (UTI) during current pregnancy (AOR: 6.4, 95\% CI, 1.95, 21.1).

CONCLUSION: Our study indicated that one among six near-term pregnant women had recto-vaginal GBS colonization. In places where universal screening is not feasible, selective screening for factors particularly history of PTD and UTI during current pregnancy may be a reasonable option. Antibiotic susceptibility testing should be performed while using Erythromycin, Clindamycin or Gentamicin.

KEYWORDS: GBS, Recto-vaginal colonization, Near-term pregnancy, Antibiotic susceptibility, Jimma, Ethiopia 


\section{INTRODUCTION}

Group B streptococcus (GBS) is a member of gram-positive streptococci. GBS, also known as Streptococcus agalactiae, is found in 15 to $40 \%$ of healthy women's colon and vagina (1-3). Maternal colonization of GBS is associated with an increased risk of urinary tract infection (UTI), endometritis, chorioamnionitis, sepsis and meningitis (3-8). Moreover, pregnant mothers may develop a premature rupture of membranes, stillbirth and low birth weight babies $(9,10)$. Obvious GBS diseases in the first week of delivery are referred to as Early-Onset Disease (EOD), whereas Late-Onset Diseases (LOD) associated with GBS happens between one week to three months after delivery (1-3).

The prevalence of vaginal colonization by GBS among pregnant women varies across the world, it has been estimated at $9.1 \%$ to $26.7 \%$ in Iran (11), and $8 \%-15 \%$ in Europe (12). The prevalence of GBS in parts of Africa such as in Egypt, Malawi, Nigeria, and Tanzania ranges from $11.3 \%$ to $23 \%$ (13-16). Prevalence of GBS recto-vaginal colonization among pregnant women has also been studied at different parts of Ethiopia including from Addis Ababa, Gondar, Adigrat, Mekele, Hawasa, and Jimma that ranges from $7.2 \%$ to $20.9 \%(17-22)$.

Universal screening of pregnant women at 35-37 weeks of gestation for GBS by rectovaginal culture is recommended so that appropriate intrapartum antibiotic prophylaxis (IAP) can be administered for positive cases (1). In the United States, since the early 1990s, medical interventions have resulted in an $80 \%$ decline in the incidence of early-onset neonatal sepsis due to GBS from 1.7 to less than 0.4 cases per 1000 live births in recent years (7). There are concerns about the emergence of drug resistance in parts of the world where IAP is practiced $(1,23$ 26). In this study's location, updated information on the trends of drug resistance on commonly used drugs is needed for practicing professionals.

Socio-demographic characteristics like greater age, increased parity, low socio-economic status, obesity level, place of residence (urban vs. rural) have been associated with increased risk of maternal colonization of GBS $(9,27)$. In Ethiopia, like other resource-limited areas, there are scanty studies conducted to assess the prevalence of recto-vaginal GBS colonization. Furthermore, screening of pregnant women for GBS is not at all routine practice. Therefore, this study assessed the prevalence of rectovaginal colonization, antibiotic susceptibility patterns and determinants of GBS isolated from near term pregnant women at Jimma University Medical Center (JUMC).

\section{MATERIALS AND METHODS}

Study setting: The study was conducted at JUMC located in the town of Jimma, $355 \mathrm{~km}$ Southwest of Addis Ababa. JUMC is the only teaching and referral hospital in the southwestern part of Ethiopia with a catchment of 15 million people. The center has bed occupancy of 650 , of which 52 beds belong to the obstetric ward. The hospital gives 24-hour service of attending normal and abnormal labor and basic and specialized antenatal care (ANC) follow-ups.

A cross-sectional study was conducted from May $1^{\text {st }}$ to August $31^{\text {st }}, 2015$.

The study population consisted of nearterm pregnant women with gestational ages of 35 to 37 weeks, who attended ANC clinic at JUMC during the study period.

Sample size: A sample size was determined using a single proportion formula with a $95 \%$ confidence level ( $Z$ of 1.96), and a standard error of 0.05 . The minimum sample size (n) was calculated by taking the carriage rate of $S$. agalactiae (GBS) in pregnant women with a $9 \%$ carriage rate from a previous study done in northern Ethiopia (18). Ten percent of the total calculated sample size $(n=126)$ was added for the non-response rate, which made the sample size 139. Among the 139 pregnant women, four refused to participate in the study. Thus, a total of one hundred thirty-five (135) pregnant women were included in the analysis yielding a $97 \%$ response rate.

Data collection: Data were collected from nearterm pregnant women through a non-probability consecutive sampling technique using a checklist within the study period by two trained midwives. Demographic characteristics and clinical outcomes of interest were collected using a wellstructured questionnaire through interview 
technique using tools from previous studies (6,7\&17-20).

For laboratory work, specimens from the lower third of the vagina and rectum were collected using sterile cotton-tipped swabs in separate sterile tubes of Ames transport medium by a trained midwife and a gynecologist. It was immediately transported to the microbiology laboratory of JUMC within one hour of collection. The specimens were inoculated directly into 5\% sheep blood agar (Oxoid Limited, Hampshire, UK) plates supplemented with $8 \mu \mathrm{g} / \mathrm{ml}$ gentamicin and $15 \mu \mathrm{g} / \mathrm{ml}$ nalidixic acid (Biomerieux, France) to prevent the growth of contaminants. The plates were incubated at $37^{\circ} \mathrm{C}$ aerobically for 24 hours. Cultures were examined for beta-hemolytic streptococci colony, and all negative plates were re-incubated for an additional 24 hours. Suspicious GBS colonies were identified by colony morphology, gram stain and biochemical tests including catalase, sodium hippurate hydrolysis, and Christie, Atkins, Munch-Petersen (CAMP) factor positivity $(4,28)$. Antimicrobial susceptibility of all GBS isolates was determined for commonly used antibiotics including Penicillin G $(10 \mu \mathrm{g})$, Ampicillin $(10 \mu \mathrm{g})$, Erythromycin $(15 \mu \mathrm{g})$, Clindamycin $(2 \mu \mathrm{g})$ and Gentamicin $(30 \mu \mathrm{g})$ by using Kirby-Bauer disk diffusion method on Mueller-Hinton agar supplemented with 5\% defibrinated sheep blood. The susceptibility results were interpreted according to the recommendations of the Clinical and Laboratory Standards Institute (CLSI) guidelines for beta hemolytic streptococci in 2012(29).

Women who were found colonized by GBS were notified by phone and told to visit the ANC clinic. Memos were produced to the attending physician indicating that intrapartum antibiotics should be given at the onset of true labor.

Statistical analysis: Collected data were used to determine associations of selected variables like socio-demographic characteristics and obstetric and medical histories with positive recto-vaginal GBS colonization using SPSS for windows version 21.0. Bivariate analysis was carried out for identified categorical variables and a $p$-value of $\leq 0.25$ was used as a cut-off point to select candidate variables for the final multiple logistic regression model. Independent predictors including history of contraception, early-onset neonatal sepsis, premature rupture of membranes, preterm delivery, UTI and other variables were determined using adjusted odds ratio with 95\% confidence interval in multiple regression analysis at $\mathrm{p}$-value $<0.05$.

\section{RESULTS}

Socio-demographic characteristics: The age of the study participants ranged from 16 to 38 years with a mean of $24.9( \pm 4.5)$ years. The majority $(62.2 \%)$ of the study participants were urban residents, married (95\%) and Muslim (68\%) by religion. Thirty-one $(23 \%)$ of them had educational level of beyond secondary school (Table 1).

Obstetric characteristics: More than half of the women $(55.6 \%)$ were multigravida and $40.7 \%$ of them were in the $37^{\text {th }}$ week of their pregnancy. Fifteen $(11.1 \%)$ of the participants had a history of preterm birth, $5.2 \%$ had premature rupture of membranes (PROM), and only $3 \%$ had neonates with previous early-onset neonatal sepsis (EONS). Contraception use among the study subjects was $65.2 \%$ (Table 2).

Medical characteristics: In our study, $21 \%$ of the study participants had history of UTI during the current pregnancy. Only three participants had a history of sexually transmitted infections (STI). Testing for human immunodeficiency virus (HIV) was done for all participants, and four women were found to be seropositive. Eight women had a history of hypertension (HTN) (Table 2). 
Table 1: Socio-demographic characteristics of near-term pregnant women involved in the study at JUMC, Jimma, Ethiopia from May to August, 2015 ( $\mathrm{n}=135)$.

\begin{tabular}{|c|c|c|}
\hline Sociodemographic factor & Frequency & Percentage \\
\hline \multicolumn{3}{|l|}{ Age group } \\
\hline Mean age & $24.9( \pm 4.5)$ years & \\
\hline $15-19$ years & 8 & $5.9 \%$ \\
\hline $20-24$ years & 57 & $42.2 \%$ \\
\hline 25-29 years & 47 & $34.8 \%$ \\
\hline 30-34 years & 16 & $11.9 \%$ \\
\hline$\geq 35$ years & 7 & $5.2 \%$ \\
\hline \multicolumn{3}{|l|}{ Residential location } \\
\hline Urban & 84 & $62.2 \%$ \\
\hline Rural & 51 & $37.8 \%$ \\
\hline \multicolumn{3}{|l|}{ Religion } \\
\hline Muslim & 91 & $67.5 \%$ \\
\hline Orthodox & 31 & $22.9 \%$ \\
\hline Protestants & 13 & $9.6 \%$ \\
\hline \multicolumn{3}{|l|}{ Educational status } \\
\hline No formal education & 28 & $20.7 \%$ \\
\hline Primary / Secondary & 76 & $56.3 \%$ \\
\hline Post-secondary & 31 & $23 \%$ \\
\hline \multicolumn{3}{|l|}{ Monthly income } \\
\hline Mean income & 2050.6( \pm 1145.6$)$ Birr & \\
\hline$<1800$ Birr & 39 & $28.8 \%$ \\
\hline 1800-2400 Birr & 52 & $38.5 \%$ \\
\hline$>2400$ Birr & 44 & $32.6 \%$ \\
\hline \multicolumn{3}{|l|}{ Marital status } \\
\hline Married & 128 & $94.8 \%$ \\
\hline Single & 7 & $5.2 \%$ \\
\hline
\end{tabular}

Table 2: Obstetric and medical characteristics of near-term pregnant women involved in the study at JUMC, Jimma, Ethiopia from May to August, 2015 ( $\mathrm{n}=135$ ).

\begin{tabular}{lcc}
\hline Obstetric variables & Frequency & Percentage \\
\hline Gravidity & 60 & \\
1 & 63 & $44.4 \%$ \\
$2-4$ & 12 & $46.7 \%$ \\
$\geq 5$ & & $8.9 \%$ \\
Gestational age & 51 & \\
$\quad 35$ weeks & 29 & $37.8 \%$ \\
$\quad 36$ weeks & 55 & $21.5 \%$ \\
$\quad 13$ weeks & 13 & $40.7 \%$ \\
Previous ANC visit & 7 & $97 \%$ \\
History of PTD & 4 & $17.3 \%$ \\
History of PROM & 88 & $5.2 \%$ \\
History of new-born with EONS & 28 & $3 \%$ \\
Contraception use & 3 & $65.2 \%$ \\
UTI during current pregnancy & 4 & $21 \%$ \\
STI during current pregnancy & $2 \%$ \\
HIV infection & 8 & $3 \%$ \\
\hline Hypertension & & $6 \%$ \\
\hline
\end{tabular}

JUMC: Jimma University Medical Center; ANC: Antenatal care; PTD: Preterm delivery; PROM: Premature rupture of membranes; EONS: Early onset neonatal sepsis; UTI: Urinary tract infection; STI: sexually transmitted infections; HIV: Human immune deficiency virus 
Prevalence of Group B Streptococci: The prevalence of GBS colonization among the study subjects was $16.3 \%(22 / 135)$. Of all the GBS isolates identified, nearly half $[45 \%$
$(10 / 22)]$ of them were recovered from the rectum only. In $9 / 22(41 \%)$ of the study subjects, GBS isolates were found from both rectum and vagina (Figure 1).

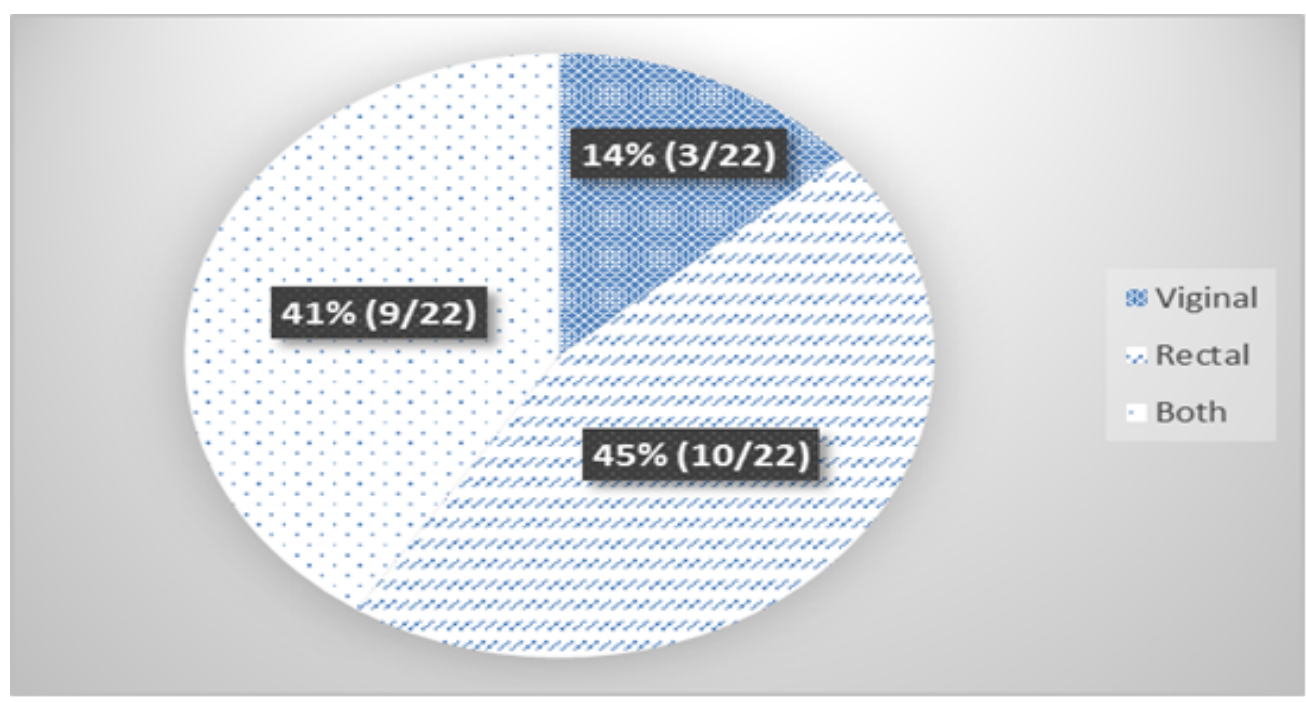

Figure 1: Site of recovery of GBS colonization among pregnant women attending $A N C J U M C$, Jimma, Ethiopia from May to August, 2015 (n=22)

Antimicrobial susceptibility testing: Twentyone $(95.5 \%)$ and $20(90.9 \%)$ of the 22 GBS isolates were susceptible to Ampicillin and Penicillin G, respectively. Only two isolates (9\%) were found to be susceptible to Clindamycin. Furthermore, intermediate susceptibility was also detected against Erythromycin in one isolate $(4.5 \%)$, and Clindamycin in three isolates $(13.6 \%)$. However, eleven $(50 \%)$ and nine $(40.9 \%)$ isolates were resistant to Erythromycin and Clindamycin, respectively. All of the isolates showed resistance to Gentamicin (100\%) (Table $3)$.

Table 3: Antimicrobial susceptibility pattern of GBS isolated from near term pregnant women at JUMC, Jimma, Ethiopia from May to August, 2015 ( $\mathrm{n}=135)$.

\begin{tabular}{lcccc}
\hline Antibiotics & Disc potency $(\boldsymbol{\mu g})$ & Susceptible & Intermediate & Resistance \\
\hline Ampicillin & 10 & $21(95.5 \%)$ & - & $1(4.5 \%)$ \\
Penicillin G & $10 \mathrm{U}$ & $20(90.9 \%)$ & - & $2(9.1 \%)$ \\
Erythromycin & 15 & $10(45.5 \%)$ & $3(13.6 \%)$ & $11(50 \%)$ \\
Clindamycin & 2 & $10(45.5 \%)$ & $1(4.5 \%)$ & $9(40.9 \%)$ \\
Gentamicin & 30 & - & - & $22(100 \%)$ \\
\hline
\end{tabular}

GBS Colonization or its absence among near term pregnant women: In univariate analysis, completing primary or secondary education, history of preterm delivery (PTD) and history of UTI during current pregnancy were significantly associated with recto-vaginal GBS colonization, while the other socio-demographic, obstetric and medical characteristics were not significantly associated with recto-vaginal GBS colonization (Table 4).

Predictors of recto-vaginal GBS colonization: Results from the multiple logistic regression analysis are presented in Table 4. Adjusting for socio-demographics, obstetric and medical 
characteristics, history of PTD and UTI during current pregnancy were significantly associated with GBS colonization. Women with previous preterm delivery were 6.3 times more likely to be colonized compared to those women with no history (AOR: 6.3, 95\% CI: 1.42, 28.3). Women with history of UTI during current pregnancy were 6.4 times more likely to be colonized by GBS (AOR: 6.4, 95\% CI: 1.95, 21.1) compared to women with no such history. Completing primary or secondary school and being employee were not associated with GBS.

Table 4: Determinants of GBS colonization among near term pregnant women at JUMC, Jimma, Ethiopia from May to August, 2015 ( $\mathrm{n}=135)$.

\begin{tabular}{|c|c|c|c|c|}
\hline \multirow[t]{2}{*}{ Variables } & \multicolumn{2}{|c|}{ GBS } & \multirow[t]{2}{*}{ COR:95\%,CI } & \multirow[t]{2}{*}{ AOR:95\%,CI } \\
\hline & $\begin{array}{l}\text { Positive } \\
(\mathrm{N}=\mathbf{2 2})\end{array}$ & $\begin{array}{l}\text { Negative } \\
(\mathrm{N}=113)\end{array}$ & & \\
\hline \multicolumn{5}{|l|}{ Age group } \\
\hline$\leq 24$ years & $13(20 \%)$ & $52(80 \%)$ & $1.14(0.34-3.85)$ & $2.5(0.23-27.3)$ \\
\hline 25-29 years & $5(10.6 \%)$ & $42(89.4 \%)$ & $0.54(0.13-2.18)$ & $0.15(0.01-2.65)$ \\
\hline$\geq 30$ years & $4(17.4 \%)$ & $19(82.6 \%)$ & $1^{*}$ & $1^{*}$ \\
\hline \multicolumn{5}{|l|}{ Residential location } \\
\hline Urban & $14(16.7 \%)$ & $70(83.3 \%)$ & $1.34(0.52-3.46)$ & $2.4(0.30-19.6)$ \\
\hline Rural & $8(15.6 \%)$ & $43(84.4 \%)$ & $1^{*}$ & $1 *$ \\
\hline \multicolumn{5}{|l|}{ Educational status } \\
\hline No formal education & $6(21.4 \%)$ & $22(78.6 \%)$ & $0.67(0.21-2.15)$ & $1.471(0.25-8.78)$ \\
\hline $\begin{array}{l}\text { Primary or secondary } \\
\text { education }\end{array}$ & $7(9.2 \%)$ & $69(90.8 \%)$ & $0.25(0.08-0.73)$ & $0.53(0.11-2.52)$ \\
\hline Post-secondary education & $9(29 \%)$ & $22(71 \%)$ & $1^{*}$ & $1^{*}$ \\
\hline \multicolumn{5}{|l|}{ Marital status } \\
\hline Married & $20(15.6 \%)$ & $108(84.4 \%)$ & $0.47(0.09-2.27)$ & $0.65(0.02-17.8)$ \\
\hline Single & $2(28.6 \%)$ & $5(71.4 \%)$ & $1 *$ & $1^{*}$ \\
\hline \multicolumn{5}{|l|}{ Gravidity } \\
\hline 1 & $10(16.7 \%)$ & $50(82.3 \%)$ & $1.19(0.35-4.0)$ & $4.14(0.33-52.3)$ \\
\hline $2-4$ & $10(15.8 \%)$ & $53(84.2 \%)$ & $0.57(0.14-2.29)$ & $1.7(0.18-16.2)$ \\
\hline$\geq 5$ & $2(16.6 \%)$ & $10(83.4 \%)$ & $1^{*}$ & $1 *$ \\
\hline \multicolumn{5}{|l|}{$\begin{array}{l}\text { History of } \\
\text { contraception }\end{array}$} \\
\hline Yes & $16(18.2 \%)$ & $72(81.8 \%)$ & $1.52(0.52-4.15)$ & $1.11(0.28-4.39)$ \\
\hline No & $6(12.8 \%)$ & $41(87.2 \%)$ & $1 *$ & $1^{*}$ \\
\hline \multicolumn{5}{|l|}{ History of PTD } \\
\hline Yes & $6(40 \%)$ & $9(60 \%)$ & $4.33(1.35-13.81)$ & $6.35(1.42-28.35) \dagger$ \\
\hline No & $16(13.3 \%)$ & $04(86.7 \%)$ & $1^{*}$ & $1^{*}$ \\
\hline \multicolumn{5}{|l|}{ History of PROM } \\
\hline Yes & $2(28.5 \%)$ & $5(71.5 \%)$ & $2.0(1.41-9.82)$ & $3.32(0.41-26.9)$ \\
\hline No & $20(15.6 \%)$ & $108(84.4 \%)$ & $1 *$ & $1^{*}$ \\
\hline \multicolumn{5}{|l|}{$\begin{array}{l}\text { History of UTI during } \\
\text { current pregnancy }\end{array}$} \\
\hline Yes & $11(39.3 \%)$ & $17(60.7 \%)$ & $5.6(2.11-15.1)$ & $6.4(1.95-21.1) \dagger$ \\
\hline No & $11(10.2 \%)$ & $96(89.8 \%)$ & $1 *$ & $1 *$ \\
\hline
\end{tabular}

N.B. $1 *$ - reference, $\dagger$ statistically significant at P-Value $<0.05$, COR- Crude Odds Ratio, AOR- Adjusted Odds Ratio 


\section{DISCUSSION}

Our study examined the prevalence as well as predictors of colonization, and antibiotic susceptibility of GBS isolates among near term pregnant women in Southwest Ethiopia. Our study showed a $16.3 \%$ prevalence of GBS rectovaginal colonization. This finding is in the range of the USA prevalence reports of $15-40 \%$ (3) and other studies done in parts of Africa: Egypt: $17.89 \%$ (15), Malawi: $16.5 \%$ (14), Nigeria: $11.3 \%$ (13), Tanzania: 23\% (16), and Democratic Republic of Congo (DRC): 20\% (29). The result is also comparable to most of the studies done in Ethiopia with a prevalence range of $11.3 \%$ $20.9 \%$ (19-22). However, a lower prevalence of recto-vaginal colonization was reported in south India: $2.3 \%$ (30), Mozambique: 1.8\% (31), and two Ethiopian studies done earlier with $7.2 \%$ in Addis Ababa (17) and 9\% in Gondar (18). The variation in prevalence at different places could be due to differences in sample size and study period, as our study was a cross-sectional study during a shorter period. Additionally, there is variation in the GBS colonization rate when sampled from rectal and/or vaginal sites of the body, and identification of GBS from clinical specimens varies from one study to another.

Identification of risk factors associated with GBS colonization has a paramount effect in reducing maternal and neonatal complications in resource-limited countries like Ethiopia, where routine screening is not the practice (32). In this study, risk factors associated with GBS colonization were history of preterm birth and UTI during the current pregnancy. History of both PTD and UTI during the current pregnancies were associated with six-fold higher odds of GBS colonization. Similar findings have been reported in a study done in the DRC (29). In women with history of PTD or UTI, it is several times as likely to have GBS colonization compared to those who don't have either history. In this instance, GBS colonization is one of the agents associated with PTD or UTI.
In our study, the majority of the isolates were susceptible to Ampicillin (95.5\%) and Penicillin $\mathrm{G}(90.9 \%)$, which is different from most studies conducted previously in which all isolates were susceptible to these two drugs $(16,20-22,33)$. The United States of America Centers for Disease Control and prevention (CDC) recommends that penicillin remains the agent of choice for IAP, with Ampicillin as an acceptable alternative to prevent GBS colonization and infection (7). This study claims that, considering the observed low rates of GBS resistance to penicillins, it is mandatory to extend the study on the GBS colonization rate among near term pregnant women with a larger number of study participants over a longer period of time. Besides, since there is no treatment guideline in this study location, it would be logical to consider GBS culture and susceptibility testing routinely to select for effective antimicrobial agents.

According to the USA CDC guidelines, penicillin-allergic women at high risk for anaphylaxis ought to receive Clindamycin if their GBS isolate is susceptible to Clindamycin and Erythromycin (7). In this study, half of the isolates were resistant to Erythromycin. This is consistent with reports from other studies done in Nigeria, 35.3\% (13), and Palestine, 43\% (33). The findings of this study also indicated that $40.9 \%$ of the isolates were resistant to Clindamycin. Gentamicin, on the other hand, showed $100 \%$ resistance to GBS isolates in our study that has been used in combination with penicillin for severe GBS associated infections (34). These increased rates of GBS resistance to Erythromycin, Clindamycin, and Gentamicin highlight the importance of mandatory antimicrobial susceptibility testing in all GBS isolated from pregnant women.

The strengths of this study are the more valid method used to identify GBS colonization, which is culture, and the prospective nature of data collection. However, this study is limited given the fact that it is a cross-sectional study to establish temporality between risk factors and GBS colonization. Moreover, since the participants were approached in a health facility, the findings may not be generalizable to the target population. 
In conclusion, the prevalence of GBS rectovaginal colonization among near-term pregnant women is comparable to most of the studies done in Ethiopia and most parts of the world. In resource-limited areas, where universal screening is not feasible, selective screening for the identified predictors may be a reasonable option. Further study to identify risk factors for rectovaginal colonization at community level is recommended. Antibiotic susceptibility of the bacterium to Ampicillin and Penicillin $G$ was high. The increased resistance rates to both Erythromycin and Clindamycin emphasize that antibiotic susceptibility tests should be done in women with allergy to penicillin. GBS is resistant to Gentamycin; reconsideration must occur before using this drug for GBS related infections until patient specific culture and antibiotic profile is known.

\section{ACKNOWLEDGMENTS}

We would like to acknowledge all the participants in this study. We also acknowledge Jimma University Institute of Health for giving us the grant to conduct this research. Pre-Publication Support Service (PREPSS) supported the development of this article by providing prepublication peer-review and copy editing.

\section{REFERENCES}

1. Schrag S, Gorwitz R, Fultz-Butts K, Schuchat A. Prevention of perinatal group B streptococcal disease. Revised guidelines from CDC.MMWR Recomm Rep 2002; 51:1- 22.

2. Nandyal RR. Update on group B streptococcal infections: perinatal and neonatal periods. $J$ Perinat Neonatal Nurs. 2008; 22(3):230-7.

3. Centers for Disease Control: Prevention of perinatal group B Streptococcal disease: a public health perspective. MMWR Recomm Rep 1996, 45(RR-7):1-24.

4. Patrick RM, Ken SR, George SK, Michael AP: Medical Microbiology. Volume 23 fourth edition. Philadelphia: Churchill Livingstone; 2002:192-235.
5. Phares CR, Lynfield R, Farley MM, MohleBoetani J, Harrison LH, Petit S, etal. Epidemiology of invasive group B streptococcal disease in the United States, 1999-2005.JAMA. 2008; 299(17):2056-65.

6. Fatemi F, Chamani L, Pakzad P, Zeraati H, Rabbani H, Asgari S. Colonization rate of group B Streptococcus (GBS) in pregnant women using GBS agar medium. Acta Medica Iranica. 2009; 47(1):25-30.

7. Verani JR, McGee L, Schrag SJ. Prevention of perinatal group B streptococcal diseaserevised guidelines from CDC, 2010.Division of bacterial diseases, National Center for Immunization and Respiratory Diseases, Centers for Disease Control and Prevention (CDC) MMWR Recommendations and reports 2010; 59(RR-10):1-36.

8. Pass MA, Gray BM, Dillon HC Jr. Puerperal and perinatal infection with Group B Streptococci. Am J Obstet Gynecol. 1982; 143:147-52.

9. Regan JA, Klebanoff MA, Nugent RP, et al. Colonization with group B streptococci in pregnancy and adverse outcome. VIP Study Group. Am J ObstetGynecol 1996; 174:1354-60.

10. Regan JA, Klebanoff MA, Nugent RP. The epidemiology of group B streptococcal colonization in pregnancy.Vaginal Infections and Prematurity Study Group.ObstetGynecol 1991; 77: 604-610.

11. Javanmanesh F, Eshraghi N. Prevalence of positive recto-vaginal culture for Group B streptococcus in pregnant women at 35-37 weeks of gestation. Med J Islam Repub Iran 2013; 27(1):7-11. pmid:23483827; PubMed Central PMCID: PMC3592944.

12. Stoll BJ and Schuchat A. Maternal carriage of group B streptococci in developing countries. Pediatric infectious disease journal.1998, 17(6): 499-503.

13. Onipede A, Adefusi O, Adeyemi A, Adejuyigbe E, Oyelese A, Ogunniyi T. Group B Streptococcus Carriage during Late Pregnancy in Ile-Ife, Nigeria.African Journal of Clinical and Experimental Microbiology. 2012; 13(3):135-43. 
14. Zoella T, Komolafe O, Igbigbi A. Prevalence of group B Streptococcus colonization in antenatal women at the Queen Elizabeth Central Hospital, Blantyre-a preliminary study. Malawi Medical Journal. 2006; 17(3):97-9.

15. Elbaradie SM, Mahmoud M, Farid M. Maternal and neonatal screening for Group B streptococci by SCP B gene-based PCR: a preliminary study. Indian $\mathrm{J}$ Med Microbiol.2009;27 (1):17-21.

16. Joachim A, Matee MI, Massawe FA, Lyamuya EF. Maternal and neonatal colonisation of group B streptococcus at Muhimbili National Hospital in Dar es Salaam, Tanzania: prevalence, risk factors and antimicrobial resistance. BMC Public Health.2009; 9:437.

17. LakewZw, Gebreegziabher TT, Teklu SM, Yigeremu MG. The prevalence of Group B Streptococus recto-vaginal colonization and antimicrobial susceptibility pattern in pregnant mothers at two hospitals of Addis Ababa, Ethiopia. Rep Health. 2014; 11:80.

18. Schmidt J, Halle E, Halle H, Mohammed T, Gunther E. Colonization of pregnant women and their newborn infants with group B streptococci in the Gondar College of Medical Sciences. Ethiop Med J. 1989; 27(3):115-9.

19. Gebremeskel TK, Zeleke TA, Mihret A, Tikue MD. Prevalence and Antibiotic Susceptibility Pattern of Streptococcus agalactiae Among Pregnant Women at Adigrat Zonal Hospital and Adigrat Health Center, Tigray, Ethiopia, Journal of Gynecology and Obstetrics. Vol. 3, No. 2, 2015, pp. 29-35. doi: 10.11648/j.jgo.20150302.

20. Alemseged $G$, Niguse $S$, Hailekiros $H$, Abdulkadir M, Saravanan M, Asmelash T. Isolation and anti-microbial susceptibility pattern of group B Streptococcus among pregnant women attending antenatal clinics in Ayder Referral Hospital and Mekelle Health Center, Mekelle, Northern Ethiopia. BMC Res Notes.2015; 8:518.
21. Mohammed M, Asrat D, Woldeamanuel Y, Demissie A. Prevalence of group B Streptococcuscolonization among pregnant women attending antenatal clinic of Hawassa Health Center, Hawassa, Ethiopia. Ethiop J Health Dev. 2012; 26:36-42.

22. Mengist A, Kannan $H$, Abdissa A. Prevalence and antimicrobial susceptibility pattern of anorectal and vaginal group B Streptococci isolates among pregnant women in Jimma, Ethiopia. BMC Res Notes 2016; 9: 351-355.

23. Apgar BS, Greenberg G, Yen G. Prevention of group B streptococcal disease in the newborn. Am Fam Physician. 2005; 71:90310.

24. Kimura K, Suzuki S, Wachino J, et al. First molecular characterization of group B streptococci with reduced penicillin susceptibility, Antimicrob Agents Chemother, 2008; 52, pp. 2890-2897

25. Back EE, O'Grady EJ, Back JD. High rates of perinatal group B Streptococcus Clindamycin and Erythromycin resistance in an upstate New York hospital. Antimicrob Agents Chemother.2012; 56: 739-742.

26. Lua B, Lib D, Cuib Y, Suia W, Huangc L, Lua X. Epidemiology of Group B streptococcus isolated from pregnant women in Beijing, China. ClinMicrobiol Infect. 2014; 20 (6): O370-O373.

27. Kleweis SM, Cahill AG, Odibo AO, Tuuli MG. Maternal Obesity and Rectovaginal Group B Streptococcus Colonization at Term.Infect Dis Obstet Gynecol. 2015; 2015:586767.

28. Nomura ML, PassiniJúnr $\quad$ R, Oliveira UM.Selective versus non-selective culture medium for group B streptococcus detection in pregnancies complicated by preterm labor or preterm-premature rupture of membranes.Braz J Infect Dis. 2006;10(4):247-50.

29. Clinical Laboratory Standards Institute (CLSI). Performance standards for antimicrobial susceptibility testing.M100S22, M2-7 and M-7, Wayne, PA. 2012. Guidelines for streptococcus spp, $\beta$ hemolytic group 2012. 
30. Mitima KT, Ntamako S, Birindwa AM, Mukanire N, Kivukuto JM, Tsongo K, Mubagwa K.: Prevalence of colonization by Streptococcus agalactiae among pregnant women in Bukavu, Democratic Republic of the Congo. J Infect DevCtries. 2014; Sep 12; 8(9):1195-200.

31. Sharmila V, Joseph NM, ArunBabu T, Chaturvedula L, Sistla S: Genital tract group B Streptococcal colonization in pregnant women: a South Indian perspective. J Infect DevCtries 2011, 5(8):592-5.

32. De Steenwinkel FD, Tak HV, Muller AE, Nouwen JL, Oostvogel PM, Mocumbi SM. Low carriage rate of group B streptococcus in pregnant women in Maputo, Mozambique. Tropical medicine \& international health: TM \& IH. 2008; 13(3):427-9.

33. American College of $\mathrm{O}$, Gynecologists Committee on Obstetric P. ACOG Committee Opinion No. 485: Prevention of early-onset group B streptococcal disease in newborns. Obstetrics and gynecology. 2011; 117 (4):1019-27.

34. Elmanama AA. Prevalence of Group B Streptococcus Colonization among Pregnant Women in Gaza strip, Palestine. 2017;25(3):1-12.

35. Liddy H, Holliman R. Group B Streptococcus highly resistant to gentamicin. J Antimicrob Chemoth. 2002; 50(1): $142-143$.

https://doi.org/10.1093/jac/dkf090 